\title{
LOS ADJETIVOS EN LAS UNIDADES TERMINOLÓGICAS POLILÉXICAS: UN ANÁLISIS MORFOSEMÁNTICO
}

\author{
Rosa Estopà
}

RESUMO: Les adjectifs peuvent prendre plusieurs valeurs suivant le nom qu'ils accompagnent. Les adjectifs qui sont partie d'unités terminologiques créent toujours une catégorie hyponymique de la catégorie du noyau nominal qu'ils déterminent. Dans cet article, nous nous proposons, premièrement, de montrer la valeur classificatrice de l'adjectif dans les unités terminologiques ayant une structure $\left[\mathrm{N}[\mathrm{A}]_{\mathrm{SAdj}}\right]_{\mathrm{SN}}$. Et, deuxiènement, d'observer comment, dans un domaine de connaissance spécialisée, on produit une sélection des adjectifs étant donné que seuls quelques adjectifs reliés à certaines catégories de nom sont pertinents à former des unités terminologiques.

PALAVRAS-CHAVE: terminologia, unidade terminológica poliléxica, sintagma terminológico, adjetivo, termo e semântica, aplicações terminológicas.

\begin{abstract}
Dependiendo de la naturaleza del núcleo, el determinante sirve para especificar con mayor detalle, indicar un fin, los medios mediante los cuales se lleva a cabo una operación, el objeto al que se aplica un proceso, o el tiempo, el lugar u otras circunstancias que llegan a convertirse, de este, en un rasgo distintivo integral del nuevo concepto. (SAGER, 1993, p.122)
\end{abstract}

El reconocimiento de las unidades de un texto con significado especializado conocido como vaciado terminológico es una de las fases básicas del trabajo en terminología. Pero si bien es una tarea central de toda aplicación terminológica, al mismo tiempo no es una tarea nada simple, sino que requiere mucho tiempo y sistematicidad en la aplicación de criterios. Es, de hecho, una de las fases más fatigosas y largas — sobre todo cuando se manipulan volúmenes de

Rosa Estopà e professora da Facultat de Traducción i Interpretació e membro do Grupo IULATERM do Institut Universitari de Lingüística Aplicada da Universitat Pompeu Fabra, Barcelona. 
información importantes- que tiene el riesgo de convertirse en poco sistemática y, por consiguiente, ineficaz.

Con la función de automatizar esta fase del trabajo terminológico, a finales de la década de los ochenta, se concibió en Canadá el primer extractor automático de terminología (TERMINO, 1988):

"Parmi toutes les tâches qui composent le travail terminologique, il en est une qui exige du terminologue beaucoup de son temps, de son attention et de ses efforts; il s'agit du dépouillement, cette étape au cours de laquelle le terminologue relève, dans le corpus parfois volumineux qu'il a constitué, les termes du domaine à l'étude et les données s'y rapportant dont il aura besoin lors de l'étape de l'analyse terminologique. Bien que, lorsqu'il dépouille les textes qu'il a rassemblés, le terminologue doive constamment user de ses connaissances et de son expérience, particulièrement lorsqu'il doit relever et découper les termes qu'il juge pertinents à son domaine de recherche, il n'en demeure pas moins que cette partie du travail terminologique, si essentielle soit-elle, présente un aspect fastidieux: que de textes il lui faut parcourir pour dépister les termes, trouver des contextes; que de fiches il doit remplir sur lesquelles doivent être consignées un nombre important de données." (PERRON, 1988, p. 24).

Con el uso de los extractores se pretende, pues, que el trabajo terminológico gane en rapidez y sistematicidad. Actualmente, disponemos de varios extractores de candidatos a unidades terminológicas (UT) basados en estrategias de diversa naturaleza. Una de las características de los extractores de naturaleza lingüística es que se centran en la detección de las UT poliléxicas, pues son las unidades más prototípicas y las más frecuentes de los textos especializados, y además son las que presentan características morfosintácticas más explícitas que facilitan su extracción.

Después de quince años de investigación en este ámbito, se puede afirmar que estos sistemas son realmente una ayuda para el profesional. Pero también se debe decir que la discriminación de las UT, e incluso de las poliléxicas, no está totalmente resuelta. Todos los extractores existentes aún presentan importantes problemas de precisión y exhaustividad. Y así suelen silenciar unidades monoléxicas que también tienen significado especializado en el texto; pero sobre todo estos sistemas proponen como candidatos a UT segmentos que no lo son, es decir que generan ruido. Se estima que entre el $45 \%$ y el $75 \%$ de los candidatos se debe rechazar ${ }^{1}$.

La causa de este cantidad tan elevada de ruido se encuentra en la estrategia de reconocimiento de UT que utilizan basada en patrones morfosintácticos, patrones que no son exclusivos de las $U^{2}{ }^{2}$. En consecuencia, el

\footnotetext{
${ }^{1}$ Para un análisis más detallado de los extractores ver Cabré, Estopà, Vivaldi, 2000.

${ }^{2}$ Analizando un corpus de textos de medicina, por ejemplo, encontramos que todos los siguientes tipos de unidades pueden presentar patrones estructurales idénticos: unidades terminológicas (médula ósea, meningitis bacteriana, enfermedad de Brill-Zinsser, prueba de la inmunoperoxidasa, sistema 234
} 
reconocimiento de estas unidades, exclusivamente a partir de patrones morfosintácticos, constituye un filtro demasiado permisivo.

Para conseguir más restrictividad es necesario proponer otras técnicas de reducción del ruido que filtren los segmentos discursivos que presentan estructuras análogas. Con esta lógica, algunos autores ya utilizan estrategias que complementan los filtros estructurales, pero que todavía no son lo suficientemente discriminantes: estrategias estadísticas (BOURIGAULT, 1993), estrategias sintácticas (JACQUEMIN, 1994), estrategias semánticas (NAULLEAU, 1998), estrategias contextuales (PEARSON, 1998).

Nuestra propuesta consiste en utilizar varias estrategias de diferente naturaleza que, combinadas, eliminen las unidades discursivas y faciliten la distinción entre UT, unidades fraseólogicas especializadas y combinaciones recurrentes con una estructura morfosintáctica aparentemente idéntica. En este artículo nos centraremos en el sintagma más frecuente de todos los dominios especializados: $\left[\mathrm{N}[\mathrm{A}]_{\mathrm{SAdj}}\right]_{\mathrm{SN}} \mathrm{y}$, en concreto, nos proponemos mostrar como es el valor del componente adjetivo el que permite discriminar las UT que presentan esta estructura.

\section{CARACTERÍSTICAS LINGÜÍSTICAS DEL ADJETIVO}

La naturaleza del adjetivo es muy compleja porque, como es sabido, participa de dos categorías gramaticales: el nombre y el verbo. Desde un punto de vista morfológico, los adjetivos tienen similitudes con los substantivos, pero, desde una perspectiva sintáctica, se parecen a los verbos. Si el adjetivo comparte unas características con los nombres y otras con los verbos es lógico preguntarse la cuestión que se plantea Wierzbicka (1986) después de repasar críticamente diferentes posiciones teóricas What is, the "raison d'être of adjectives as special word class?.

Esta autora piensa que lo original del adjetivo respecto del verbo y del sustantivo es la atribución. Para Wierzbicka, el elemento que caracteriza el adjetivo, desde el punto de vista semántico, es que añade un rasgo al sustantivo que acompaña. Soler (1997, p. 61), en una tesis sobre la representación de los desajustamientos [ $\mathrm{N}+$ Adj], intenta justamente establecer los diversos valores con los que un adjetivo puede modificar un nombre.

Existen criterios de muy diversa naturaleza para caracterizar el adjetivo, por bien que, desde el punto de vista semántico, se ha tendido a diferenciar los adjetivos entre adjetivos relacionales y adjetivos calificativos. El hecho que,

mononuclear fagocítico, etc.); unidades fraseológicas especializadas (acumulación de líquido extravascular, aumento de la permeabilidad vascular, etc.); combinaciones especializadas recurrentes (radiografia de la mano, masaje en las cervicales, etc.); unidades discursivas (aumento del número de casos, décadas de los años treinta, distribución geográfica, manera específica, color rojizos, matadero de Brisbane, etc.); y unidades léxicas terminológicamente no pertinentes para el ámbito temático del texto (azul de metileno, trabajadoras sociales, condiciones de vida, clase social, etc.). 
tradicionalmente, se hayan caracterizado los adjetivos como palabras que denotan calidades o propiedades de las entidades a las que modifican, ha inducido Bosque (1993, p.10) a decir que

“(...) con bastante frecuencia se olvida que no dejan de ser adjetivos aquellos que no representan nociones. Los adjetivos que se suelen llamar "relacionales", no "predicativos", "clasificatorios", "denominales" y "referenciales", entre otras denominaciones, se caracterizan precisamente porque no son calificativos, es decir no denotan cualidades o propiedades de los sustantivos, sino por el hecho de que establecen conexiones entre esas entidades y otros dominios o ámbitos externos a ellas con las cuales sitúan o clasifican a los sustantivos sobre los que inciden".

Esta dicotomía presentada en dos polos totalmente opuestos no da cuenta de la realidad, que es mucho más ambigua, pues hay adjetivos que pueden ser clasificados como calificativos y como relacionales:

Es evidente que no podemos interpretar de igual manera el adjetivo musical cuando aparece en SN como sonido musical y cuando aparece en crítica musical. En el primer caso, musical es un adjetivo calificativo, por lo que denota una cualidad o una propiedad del sonido, pero en el segundo es relacional o clasificativo, puesto que nos habla de una clase de crítica, es decir nos introduce en un dominio (el de la música) en relación con el cual hay que entender la crítica. (BOSQUE, 1993, p.119)

Por eso, Bosque (1993) y Soler (1997), desde una perspectiva estrictamente semántica, prefieren hablar, no de tipos de adjetivos, sino de valores (relacional o calificativo) que pueden adquirir los adjetivos según el nombre con el que se combinen y el contexto y ámbito temático en el que aparecen:

Las diferencias entre los adjetivos calificativos y los adjetivos relacionales se manifiestan en la morfología, la sintaxis y el léxico e incluso una parte de ellas tiene su origen en nociones de naturaleza pragmática y existen casos de ambigüedad entre la interpretación calificativa y la interpretación relacional de un adjetivo. (BOSQUE, 1993, p. 14)

Así, desde el punto de vista terminológico, la función del adjetivo en las UT con estructura $\left[\mathrm{N}[\mathrm{A}]_{\mathrm{SAdj}}\right]_{\mathrm{SN}}$ es siempre clasificar al nombre, subespecificarlo de su clase más genérica ${ }^{3}$. Esta es una de les razones por la que asumimos la idea de Bosque que los adjetivos pueden presentar dos valores no excluyentes entre sí. Y dependerá del contexto (y del paradigma conceptual — añadimos nosotros- ) que se active un valor $u$ otro.

En esta línea, Maingueneau y Salvador (1995) describen esta misma dualidad a través de los conceptos de Milner de clasificatoriedad y noclasificatoriedad:

Emprar un adjectiu de manera classificatòria (com que hi ha adjectius mixtos i contextos molt particulars, és preferible parlar d'usos que d'adjectius

${ }^{3}$ Por ejemplo, hepatitis bacteriana es un hipónimo de hepatitis y célula gigante lo es de célula. 236 
classificatoris) equival a fer entrar els referents en classes delimitables portadores d'informació. Usar un adjectiu de manera no classificatòria és, de fet, avaluar un objecte. El significat dels adjectius no permet, en aquest darrer cas, establir categories discretes (ço és, classificar un conjunt), sinó que es vincula essencialment a l'acte d'enunciació concret on s'inscriu. (MAINGUENEAU y SALVADOR, 1995, p. 132)

En las UT, el uso activado es exclusivamente el relacional porque la función del adjetivo en este tipo de unidades especializadas es siempre clasificar el sustantivo al que se adjunta.

Los adjetivos que por defecto son relacionales se diferencian del resto de adjetivos por motivos principalmente semánticos, pero también de orden morfosintáctico. Morfológicamente, estos adjetivos suelen ser derivados, formados a partir de un nombre y un sufijo determinado (-al, -ar, -ico, -ismo, etc.). Sintácticamente, no tienen naturaleza predicativa y, por tanto: no pueden funcionar como atributos en frases copulativas, no pueden ser predicados en cláusulas reducidas, no aparecen en posición prenominal, no se pueden coordinar con adjetivos calificativos, requieren adyacencia con el sustantivo. Y semánticamente: no aceptan una modificación de gradación ${ }^{4}$, manifiestan casi todas las relaciones semánticas que permiten los complementos con de.

Aunque, como señala Bosque (1993, p. 20), estas características de los adjetivos relacionales son por defecto tendencias significativas que tienen un valor estadístico más que sistemático, ya que en dominios especializados las recategoritzaciones son muy frecuentes.

Así, los adjetivos, mayoritariamente simples, que tienden a activar el valor calificativo en tanto que unidades especializadas, no aparecen nunca aisladamente, pero sí que pueden formar parte de una UT poliléxica. Cuando esto ocurre, desactivan el valor calificativo adquirido por defecto y activan el valor relacional. Esto significa que los adjetivos calificativos pueden, en un dominio concreto, adquirir el valor de adjetivo clasificador:

Una expresión tal como trocha ancha utilizada por un ingeniero del ferrocarril se convierte en una expresión terminologizada; es un término de un lenguaje especializado mientras que en el lenguaje general no siempre se considera una forma lexicalizada.(SAGER, 1993, p. 96)

En esta misma línea Bosque (1993, p.20) cita un ejemplo del papel del sufijo -oso (considerado tradicionalmente calificativo) utilizado con valor relacional en algunas materias científico-técnicas:

Tampoco son muchos les adjetivos relacionales entre el numeroso grupo de adjetivos terminados en -oso. Entre las excepciones están los términos técnicos que se pueden usar como adjetivos relacionales, además de como adjetivos calificativos, en los casos en los que la presencia de la materia denotada por el sustantivo del que derivan puede

\footnotetext{
${ }^{4}$ Bosque nota que es lógico que los adjetivos relacionales no acepten esta propiedad porque no denotan cualidades, sino clases o individuos.
} 
ser distintiva de alguna clase natural, lo que les permite adquirir propiedades clasificatorias: glanduloso, nervioso, arenoso, oleaginoso, etc. (BOSQUE, 1993, p. 20)

De hecho, cada área de conocimiento cuenta con un conjunto de adjetivos calificativos que pueden ser constituyentes de una UT poliléxica. Y, cuando estos adjetivos forman parte de una UT poliléxica, se convierten en adjetivos clasificadores subespecificando el sustantivo al que modifican.

En medicina, por ejemplo, los adjetivos que denotan el grado de intensidad, el color o la forma, muchas veces funcionan como clasificadores: cefalalgia moderada, cefalalgia leve, cefalalgia aguda, cefalalgia intensa, cefalalgia pulsativa, edema benigno, edema maligno, etc.; sustancia blanca, sustancia gris, mancha negra, tifus amarillo, fiebre amarilla, glóbulo blanco, glóbulo rojo, etc.; cuerpo estriado, cuerpo esponjoso, pie plano, pie vacio, bronquitis fétida, meningitis purulenta, etc.

En los textos especializados, un indicio para reconocer que un adjetivo está actuando de clasificador es la presencia de adjetivos en relación de contraste que modifican a un mismo sustantivo: maxilar superior / maxilar inferior, intestino grueso / intestino delgado, paladar blando / paladar duro, yugular anterior / yugular posterior, palatino anterior / palatino posterior, pulmón izquierdo / pulmón derecho, etc.

Ni superior, inferior, grueso, delgado, blando, duro, anterior, posterior, izquierdo ni derecho, son adjetivos que, autónomamente, sean ni especializados ni clasificadores; sólo cuando se combinan con un término (que pertenece a un paradigma conceptual), lo subclasifican y convierten la combinación $\left[\mathrm{N}[\mathrm{A}]_{\mathrm{SAdj}}\right]_{\mathrm{SN}}$ en un tipo de la clase a la que pertenece su núcleo nominal. Uno de los rasgos básicos de los adjetivos relacionales es, pues, que crean una subclase de la clase a la que pertenece el nombre. $Y$ en el caso que el adjetivo de una secuencia $\left[\mathrm{N}[\mathrm{A}]_{\mathrm{SAdj}}\right]_{\mathrm{SN}}$ no funcione de clasificador significa que esta secuencia no es terminológica, sino discursiva o fraseológica.

\section{LOS ADJETIVOS PERTINENTES EN MEDICINA}

A partir de estas consideraciones hemos elaborado una clasificación de los adjetivos que en el ámbito médico pueden formar parte de una UT poliléxica con estructura $\left[\mathrm{N}[\mathrm{A}]_{\mathrm{SAdj}}\right]_{\mathrm{SN}}$. Esta tipología que proponemos tiene en cuenta las ideas expuestas en Soler (1997), Bosque (1993) y Lorente (1994). Esta clasificación permite priorizar la información específica que aportan estos adjetivos al nombre, información que sirve para discriminar semánticamente este nombre respecto de las unidades de su misma clase: 


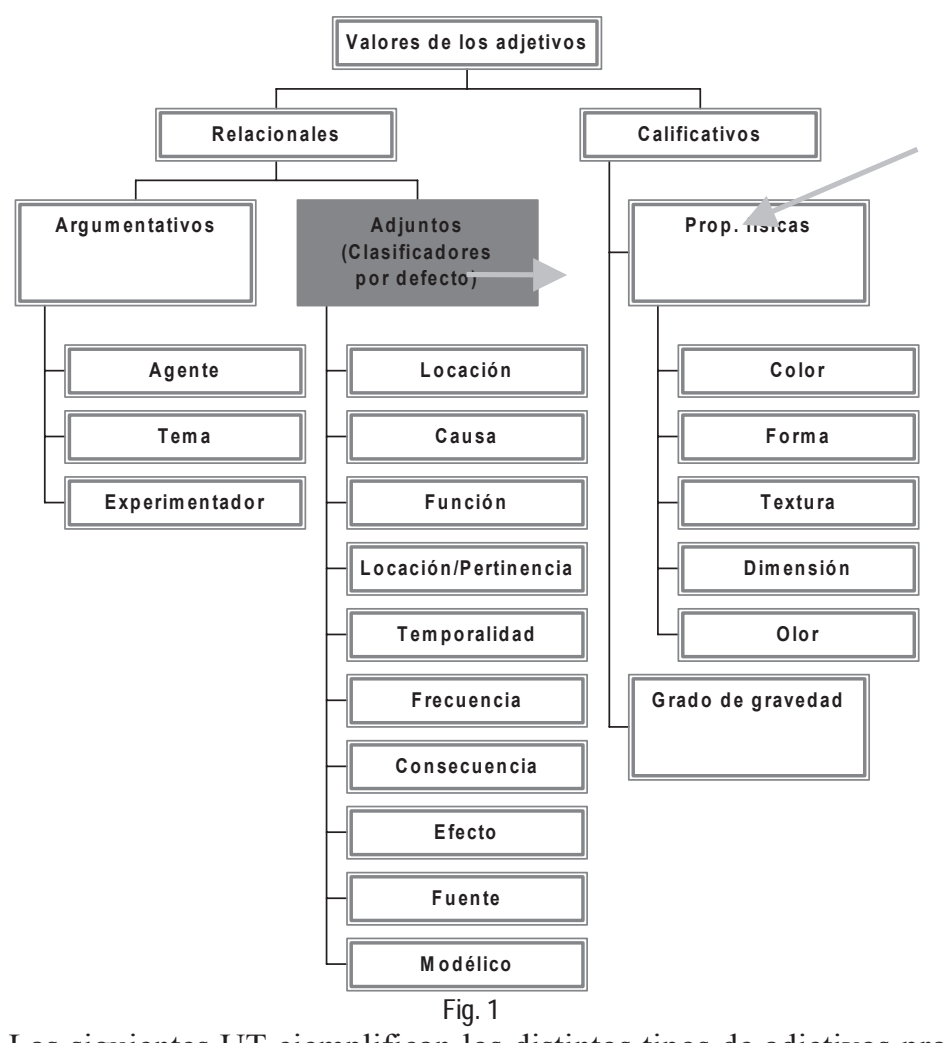

Las siguientes UT ejemplifican los distintos tipos de adjetivos propuestos en la figura superior como pertinentes en el ámbito de la medicina: solar, etc.

Adjetivos que indican el agente: neuritis alcohólica, meningitis química, ictericia

Adjetivos que indican el tema: concentración bactericida, diseminación hematogénica, infección cutánea, presión venosa, etc.

Adjetivos que indican el experimentador: demencia juvenil, demencia senil, parálisis infantil, enfermedad profesional, etc.

Adjetivos que indican la localización del $\mathrm{N}$ en el cuerpo: Componentes del cuerpo humano + sufijo relacional: embolia cerebral, embolia pulmonar, fibrosis pulmonar, trombosis cerebral, etc. No anatómico: adenopatía regional, extremidades inferiores, lóbulo superior, pulmón derecho, pulmón izquierdo, etc.

Adjetivos que indican la causa del N: enfermedad virica, fiebre tifica, fiebre reumática, taquicardia anémica, etc.

Adjetivos que indican la función del N: nervio auditivo, placa motora, sistema respiratorio, etc.

Adjetivos que indican localización / pertinencia del N: arteria alveolar, arteria bucal, cavidad nasal, nervio palatino, papila lingual, etc.

Adjetivos que indican la temporalidad o la frecuencia del $\mathrm{N}$ : conjuntivitis primaveral, diagnóstico precoz, neumonitis crónica, etc. 
Adjetivos que indican la consecuencia que ocasiona el $\mathrm{N}$ : adiposis dolorosa, lesión irritativa, taquicardia febril, etc.

Adjetivos que indican la fuente con la que funciona el $\mathrm{N}$ : análisis químico, análisis térmico, pelvimetría radiológica, radiografía electrónica, etc.

Adjetivos modélicos que indican la relación respecto de un estándar: neumonía atípica, dermatitis atópica, neumotórax patológico, pulmón artificial, etc.

Adjetivos que indican la morfología del N:

- color: escara negra, fiebre amarilla, mácula azul, sustancia gris, glóbulo rojo, etc.

- forma o composición: arteria callosa, verruga plana, diarrea acuosa, eritema nudoso, pie plano, tejido adiposo, etc.

- textura: paladar duro, paladar blando, venda elástica, etc.

- dimensión: articulación gruesa, célula gigante, intestino delgado, etc.

- olor: bronquitis fétida, meningitis purulenta, peritonitis pútrida, etc.

Adjetivos que indican el grado de gravedad del N: anemia moderada, cefalea intensa, infección secundaria, enteritis grave, diarrea leve, etc.

\section{LOS ADJETIVOS BASE DE LA DETECCIÓN DE LAS UNIDADES TERMINOLÓGICAS POLILÉXICAS: UN APLICACIÓN EN EL CAMPO DE LA MEDICINA}

La estructura $\left[\mathrm{N}[\mathrm{A}]_{\mathrm{SAdj}}\right]_{\mathrm{SN}}$ es, como decíamos, la más productiva de los textos especializados, pero a la vez es una de las que ocasiona más ruido, pues formalmente presenta una estructura que no es exclusiva de las UT. Con el objetivo de mejorar el reconocimiento de las unidades que presentan esta estructura, hemos analizado esta estructura en un corpus textual y en un corpus lexicográfico de medicina. Los resultados de este análisis muestran que el carácter especializado o discursivo de una unidad con estructura $\left[\mathrm{N}[\mathrm{A}]_{\mathrm{SAdj}}\right]_{\mathrm{SN}}$ depende de la naturaleza especializada o no del nombre y del adjetivo que la integran. En esta línea, distinguimos cuatro posibilidades:

$$
\begin{aligned}
& {\left[\mathrm{N}_{\text {esp }}\left[\mathrm{A}_{\text {esp }}\right]_{\mathrm{SAdj}}\right]_{\mathrm{SN}}=\mathrm{UT}} \\
& {\left[\mathrm{N}_{\text {no esp }}\left[\mathrm{A}_{\text {essp }}\right]_{\mathrm{SAdj}}\right]_{\mathrm{SN}}=\mathrm{UT}} \\
& {\left[\mathrm{N}_{\text {esp }}\left[\mathrm{A}_{\text {no esp }}\right]_{\mathrm{SAdj}}\right]_{\mathrm{SN}}=\mathrm{UT} \text { o UD }} \\
& {\left[\mathrm{N}_{\text {no esp }}\left[\mathrm{A}_{\text {no esp }}\right]_{\mathrm{SAdj}}\right]_{\mathrm{SN}}=\mathrm{UD} \text { o UL }}
\end{aligned}
$$

De estas posibilidades inferimos los resultados siguientes:

a) Si el núcleo de una unidad es una UT y el adjetivo que lo complementa también transmite un significado especializado, el resultado es siempre una UT: antibiótico bactericida, degeneración lenticular, dermatitis actínica, embolia cerebral, inhibición enzimática, inyección endodérmica, intervención quirúrgica, neurosis gástrica, etc.

b) Si el nombre de la unidad no es una UT, pero el adjetivo que lo clasifica es especializado, la combinación resultante es también una UT': canal coclear, capacidad pulmonar, punto alveolar, timbre nasal, vaso linfático, etc.

\footnotetext{
${ }^{5}$ Esta combinación es muy productiva cuando la UT poliléxica pertenece a la clase semántica de las
} 240 
c) Si, en cambio, el nombre es una UT y el adjetivo no es especializado, se puede tratar o de una UT (verruga plana, edema maligno, eritema simple, fiebre amarilla, laringitis aguda, nefritis local, etc.) o de una unidad discursiva (UD) (hepatitis rara, inyección fuerte, paciente diferente, reacción adversa, etc.). En este segundo caso, el sistema debería proponer como terminológicos los núcleos nominales de cada unidad discursiva y eliminar el ruido que genera el adjetivo que los modifica.

d) Finalmente, si un segmento no está formado por ninguna unidad con significado especializado, la unidad resultante es o una unidad de discurso libre (elemento clave, estudio reciente, mecanismo posible, viajero internacional) o una unidad léxica sin valor especializado en el texto (animal doméstico, continente americano, zona urbana) y, por tanto, en ninguno de los dos casos es pertinente de recoger.

Por tanto, un extractor, después de aplicar el filtro de estructuras posibles, debe saber si los componentes de estas estructuras tienen valor especializado o no. Para ello, proponemos que un extractor utilice un diccionario de UT simples, no construidas, y una herramienta de detección de formantes grecolatinos basado en un diccionario de unos mil formantes. En Estopà (1999) se observa que la mayoría de las unidades derivadas y de las compuestas en medicina están todas relacionadas o con un término simple o sobre todo con un formante grecolatino. En este sentido, es sabido que aproximadamente unos 1.100 formantes clásicos (raíces y afijos) son la base de más del $80 \%$ de las unidades de significación especializada usadas en medicina: En torno a mil raíces de procedencia griega o latina componen la casi totalidad de los términos médicos de origen clásico y de los neologismos (LÓPEZ PIÑERO y TERRADA FERRANDIS, 1990, p. 29).

Una vez establecido el carácter formalmente especializado del nombre y del adjetivo que integran el sintagma, el extractor podrá proponer como UT las que responden a las combinaciones $\left[\mathrm{N}_{\text {esp }}\left[\mathrm{A}_{\text {esp }}\right]_{\mathrm{SAdi}}\right]_{\mathrm{SN}} \mathrm{y}\left[\mathrm{N}_{\text {no esp }}\left[\mathrm{A}_{\text {esp }}\right]_{\mathrm{SAdi}}\right]_{\mathrm{SN}}, \mathrm{y}$ rechazar las que presentan la combinación $\left[\mathrm{N}_{\text {no esp }}\left[\mathrm{A}_{\text {no esp }}\right]_{\mathrm{SAdj}}\right]_{\mathrm{SN}}$.

Pero para la discriminación de las UT con estructura $\left[\mathrm{N}_{\text {term }}\left[\mathrm{A}_{\text {no esp }}\right]_{\mathrm{SAdj}}\right]_{\mathrm{SN}}$ no será suficiente con la aplicación de filtros morfosintácticos, sino que además será necesario poderlos restringir semánticamente. Por eso, proponemos unos filtros morfosemánticos que, aplicados después de los morfosintácticos, permiten identificar cuando una unidad con esta estructura es terminológica o es una combinación libre.

Antes de aplicar el filtro morfosemántico, pero, se debe establecer: a) la clase semántica a la que pertenece el $\mathrm{N}_{\text {term, }}$ b) la clase semántica del $\mathrm{A}_{\text {no esp }}$, y c) si la combinación $\left[\mathrm{N}_{\text {term }}\left[\mathrm{A}_{\text {no esp }}\right]_{\mathrm{SAdj}}\right]_{\mathrm{SN}}$ da lugar a una UT. Porque cada dominio de especialidad prioriza un grupo restringido de adjetivos que sirven para clasificar los nombres sólo en el dominio considerado. E incluso, dentro de una parcela

partes del cuerpo humano, pero el núcleo de la unidad se refiere a una parte del cuerpo que recibe el nombre de un objeto del mundo con el que mantiene un grado de semejanza. 
concreta del conocimiento especializado, no todos los adjetivos pueden acompañar a todos los nombres. Estos dos hechos implican que los filtros se deben aplicar siempre en el contexto de un dominio específico.

Por lo que se refiere al análisis de los nombres que aparecen en esta estructura en medicina y con el fin de atribuirlos a una clase semántica, el filtro que proponemos parte de la clasificación de nombres siguiente que recoge los principales clases de nombres pertinentes para transmitir el conocimiento especializado médico:

NOMBRE A: enfermedades, estados o manifestaciones patológicas

NOMBRE B: partes y componentes del cuerpo humano

NOMBRE C: objetos instrumentales

NOMBRE D: métodos, pruebas y técnicas

NOMBRE E: acciones

NOMBRE F: operaciones.

Para determinar los $A_{\text {no esp }}$ que pueden formar parte de una UT, nos basamos en la clasificación de los adjetivos pertinentes en medicina que hemos presentado en el apartado anterior, basada en las propiedades, intrínsecas o extrínsecas, que los adjetivos manifiestan respecto del nombre que complementan.

A continuación, y después de un análisis de las UT con este patrón en el corpus lexicográfico y en el corpus textual médico, podemos establecer las siguientes combinaciones de clases de nombres con clases de adjetivos terminológicamente pertinentes en este ámbito:

\begin{tabular}{|c|c|c|c|c|c|c|}
\hline \multirow[t]{2}{*}[\mathrm{N}_{\text{term}}[\mathrm{A}_{\mathrm{no}}\text{esp}]\mathrm{SAdj}]{$\mathrm{sN}=\mathrm{UT}$} & \multicolumn{6}{|c|}{ NOMBRES } \\
\hline & A & B & C & D & $E$ & $\mathbf{F}$ \\
\hline ADJ1 (LOCALIZACIÓN) & $\mathrm{X}$ & $\mathrm{X}$ & $\mathrm{X}$ & $\mathrm{X}$ & $\mathrm{X}$ & $\mathrm{X}$ \\
\hline ADJ2 (CAUSA) & $X$ & - & -- & -- & $\mathrm{X}$ & - \\
\hline ADJ3 (TIEMPO) & $x$ & -- & $\mathrm{X}$ & -- & -- & - \\
\hline ADJ4 (FRECUENCIA) & $X$ & -- & -- & $x$ & -- & -.- \\
\hline ADJ5 (EFECTO) & $\mathrm{X}$ & -- & -- & $\mathrm{X}$ & -- & -. \\
\hline ADJ6 (FUNCIÓN) & -- & $\mathrm{X}$ & $\mathrm{X}$ & $\mathrm{X}$ & $\mathrm{X}$ & $\mathrm{X}$ \\
\hline ADJ7 (FUENTE) & -- & -- & $x$ & $\mathrm{X}$ & $\mathrm{X}$ & $\mathrm{x}$ \\
\hline ADJ8 (FUNCIONAMIENTO) & $x$ & -- & $x$ & $x$ & $x$ & -- \\
\hline ADJ9 (PARTES) & -- & -- & $x$ & -- & -- & -. \\
\hline ADJ10 (MATERIA) & -- & $\mathrm{X}$ & $\mathrm{X}$ & -- & -- & - \\
\hline ADJ11 (AGENTE) & $X$ & -- & -- & -- & -- & -- \\
\hline ADJ12 (EXPERIMENTADOR) & $X$ & - & -- & -- & -- & - \\
\hline ADJ13 (CANTIDAD) & $x$ & -- & -- & -- & $X$ & $x$ \\
\hline ADJ14 (GRAVEDAD) & $X$ & -- & -- & -- & -- & -- \\
\hline ADJ15.1 (COLOR) & $X$ & $\mathrm{X}$ & -- & -- & $X$ & -- \\
\hline ADJ15.2 (TEXTURA) & $X$ & -- & -- & -- & -- & -- \\
\hline ADJ15.3 (OLOR) & $X$ & -- & -- & -- & -- & -- \\
\hline ADJ15.4 (FORMA) & $X$ & $\mathrm{X}$ & $\mathrm{X}$ & -- & -- & $\bar{x}$ \\
\hline ADJ15.5 (DIMENSIÓN) & -- & $\mathrm{X}$ & -- & -- & -- & - \\
\hline ADJ15.6 (OTROS) & $x$ & -- & $\mathrm{X}$ & -- & -- & - \\
\hline ADJ16 (ESTÁNDAR) & $\mathrm{X}$ & -- & -- & -- & -- & -- \\
\hline
\end{tabular}

Fig. 2 
A partir de las combinaciones establecidas en la figura 2 podemos formular los siguientes filtros morfosemánticos que permiten discriminar las UT pertinentes en medicina que presentan la estructura $\left[\mathrm{N}_{\text {term }}\left[\mathrm{A}_{\text {no esp }}\right]_{\mathrm{SAdj}}\right]_{\mathrm{SN}}$ :

\section{A. Nombres A:}

[NOMBRE A $\left.[\mathrm{ADJ} 1]_{\mathrm{SAdi}}\right]_{\mathrm{SN}=\mathrm{UTP}}{ }^{6}$

$\left.[\text { NOMBRE A[ADJ2 }]_{\mathrm{SAdj}}\right]_{\mathrm{SN}}=\mathrm{UTP}^{7}$

$\left.[\text { NOMBRE A[ADJ3 }]_{\mathrm{SAdj}}\right]_{\mathrm{SN}}=\mathrm{UTP}^{8}$

$\left.[\text { NOMBRE A[ADJ4 }]_{\mathrm{SAdj}}\right]_{\mathrm{SN}}=\mathrm{UTP}^{9}$

$\left.[\text { NOMBRE A[ADJ5 }]_{\mathrm{SAdj}}\right]_{\mathrm{SN}=}=\mathrm{UTP}^{10}$

$\left.[\text { NOMBRE A[ADJ } 8]_{\mathrm{SAdj}}\right]_{\mathrm{SN}}=\mathrm{UTP}^{11}$

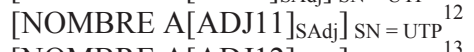

[NOMBRE A[ADJ12 $\left.]_{\mathrm{SAdj}}\right]_{\mathrm{SN}=\mathrm{UTP}}{ }_{14}^{13}$

$\left.[\text { NOMBRE A[ADJ13 }]_{\mathrm{SAdj}}\right]_{\mathrm{SN}=\mathrm{UTP}}{ }^{14}$

[NOMBRE A[ADJ14 $\left.]_{\mathrm{SAdj}}\right]_{\mathrm{SN}}=\mathrm{UTP}^{15}$

[NOMBRE A[ADJ15.1 $\left.]_{\mathrm{SAdj}}\right]_{\mathrm{SN}=\mathrm{UTP}}{ }_{17}^{16}$

$\left.[\text { NOMBRE A[ADJ15.2 }]_{\mathrm{SAdj}}\right]_{\mathrm{SN}}=\mathrm{UTP}_{18}$

$\left.[\text { NOMBRE A[ADJ15.3 }]_{\mathrm{SAdj}}\right]_{\mathrm{SN}=}=\mathrm{UTP}_{19}{ }_{19}$

$\left.[\text { NOMBRE A[ADJ15.4 }]_{\mathrm{SAdj}}\right]_{\mathrm{SN}=\mathrm{UTP}}{ }_{20}^{19}$

$\left.[\text { NOMBRE A[ADJ15.6 }]_{\mathrm{SAdj}}\right]_{\mathrm{SN}}=\mathrm{UTP}_{21}^{20}$

$\left.[\text { NOMBRE A[ADJ16 }]_{\mathrm{SAdj}}\right]_{\mathrm{SN}}=\mathrm{UTP}^{21}$

\section{B. Nombres B:}

$\left[\text { NOMBRE B }[\mathrm{ADJ} 1]_{\mathrm{SAdj}}\right]_{\mathrm{SN}}=\mathrm{UTP}_{23}^{22}$

$\left[\text { NOMBRE B }[\mathrm{ADJ} 6]_{\mathrm{SAdj}}\right]_{\mathrm{SN}=\mathrm{UTP}^{23}}$

${ }^{6}$ estafiloma anterior, estafiloma posterior, fistula externa, fistula interna, otitis externa, otitis interna, etc.

${ }^{7}$ hemoglobunuria accidental, hemorragia provocada, micosis endógena, sifilis adquirida, etc.

${ }^{8}$ catarro estival, conjuntivitis primaveral; alopecia prematura, linfodema precoz, etc.

9 artritis temporal, locura transitoria, cólera esporádico, estenosis permanente, faringitis crónica; endocarditis terminal, hematuria inicial, neumonía terminal, etc.

${ }^{10}$ meningitis silenciosa.

${ }^{11}$ endocarditis lenta, hemorragia activa, trombosis pasiva, etc.

${ }_{13}^{12}$ edema alimentario, ictericia solar, meningitis química, etc.

13 esclerosis presenil, demencia senil, demencia juvenil; dermatitis profesional, enfermedad rural, neurosis militar, etc.; fiebre española, fiebre tropical, hemoptisis oriental, meningitis africana, etc.

${ }_{14}$ epilepsia focal, epilepsia generalizada, fistula completa, fistula simple, hipermetropía total, neumonía parcial, etc.

${ }^{15}$ encefalitis secundaria, epilepsia menor, epilepsia mayor, faringitis aguda, edema maligno, edema benigno, hemorragia grave, otitis aguda, etc.

${ }^{16}$ asfixia azul, atrofia blanca, atrofia gris, atrofia roja, atrofia negra, edema azul, fiebre negra, fiebre amarilla, etc.

${ }^{17}$ estomatitis cremosa, pus cremoso, etc.

${ }^{18}$ bronquitis fétida, meningitis purulenta, peritonitis pútrida, etc.

${ }^{19}$ hemangioma plana, pitiriasis redonda, úlcera circular, etc.

${ }^{20}$ faringitis seca, hemoaglutinación caliente, nódulo frío, etc.

${ }^{21}$ dermatitis atópica, erupción anómala, estenosis falsa, mácula comuna, migraña común, etc.

${ }^{22}$ fibra endógena, fibra exógena, ventrículo lateral, ventrículo mediano, etc. (arteria espinal anterior, arteria espinal posterior, arteria pulmonar derecha, arteria pulmonar izquierda, etc.) 
$\left[\text { NOMBRE B }[\text { ADJ10 }]_{\text {SAdj }}\right]_{\mathrm{SN}=\mathrm{UTP}^{24}}$

$\left.[\text { NOMBRE B [ADJ15.1 }]_{\mathrm{SAdj}}\right]_{\mathrm{SN}}=\mathrm{UTP}^{25}$

$\left.[\text { NOMBRE B [ADJ15.5 }]_{\text {SAdj }}\right]_{\text {SN }}=\mathrm{UTP}^{26}$

$\left[\text { NOMBRE B }[\text { ADJ15.4 }]_{\text {SAdj }}\right]_{\mathrm{SN}=\mathrm{UTP}^{27}}$

C. Nombres C:

$\left[\text { NOMBRE C }[\text { ADJ } 1]_{\mathrm{SAdj}}\right]_{\mathrm{SN}=\mathrm{UTP}^{28}}$

$\left[\text { NOMBRE C }[\text { ADJ3 }]_{\text {SAdj }}\right]_{\text {SN }=\text { UTP }^{29}}$

$\left.[\text { NOMBRE C [ADJ6 }]_{\text {SAdj }}\right]_{\text {SN }}=$ UTP $^{30}$

$\left.[\text { NOMBRE C [ADJ7 }]_{\text {SAdj }}\right]_{\text {SN }}=$ UTP $^{31}$

[NOMBRE C [ADJ8 $\left.]_{\text {SAdj }}\right]_{\mathrm{SN}}=\mathrm{UTP}_{33}^{32}$

$\left.[\text { NOMBRE C [ADJ9 }]_{\text {SAdj }}\right]_{\mathrm{SN}}=\mathrm{UTP}^{33}$

$\left[\text { NOMBRE C }[\text { ADJ } 10]_{\text {SAdj }}\right]_{\text {SN }=U \mathrm{UTP}^{34}}$

$\left[\text { NOMBRE C }[\text { ADJ } 15.4]_{\text {SAdj }}\right]_{\mathrm{SN}}=\mathrm{UTP}^{35}$

$\left[\text { NOMBRE C }[\text { ADJ15.6 }]_{\text {SAdj }}\right]_{\mathrm{SN}}=\mathrm{UTP}^{36}$

\section{Nombres D:}

$\left.[\text { NOMBRE D [ADJ1 }]_{\text {SAdj }}\right]_{\mathrm{SN}=\mathrm{UTP}^{37}}{ }^{37}$

[NOMBRE D [ADJ4 $\left.]_{\text {SAdj }}\right]_{\text {SN }=\text { UTP }^{38}}$

$\left[\text { NOMBRE D }[\text { ADJ6 }]_{\text {SAdj }}\right]_{\text {SN }=U T P}{ }^{39}$

$\left.[\text { NOMBRE D [ADJ7 }]_{\mathrm{SAdj}}\right]_{\mathrm{SN}}=\mathrm{UTP}^{40}$

$\left[\text { NOMBRE D }[\text { ADJ8 }]_{\text {SAdj }}\right]_{\mathrm{SN}}=\mathrm{UTP}^{41}$

\section{E. Nombres E:}

$\left[\text { NOMBRE E }[\text { ADJ } 1]_{\text {SAdj }}\right]_{\mathrm{SN}=\mathrm{UTP}}{ }^{42}$

[NOMBRE E $\left.[\mathrm{ADJ} 2]_{\mathrm{SAdj}}\right]_{\mathrm{SN}}=\mathrm{UTP}^{43}$

$\left[\text { NOMBRE E }[\text { ADJ6 }]_{\text {SAdj }}\right]_{\text {SN }=\text { UTP }}{ }^{44}$

${ }^{23}$ hidrocefalia obstructiva, nervio motor, proteina defensiva, etc.

${ }^{24}$ fibra amarilla, fibra gris, fibra clara, fibra blanca, fibrocartílago amarillo, glóbulo rojo, etc.

${ }^{25}$ célula poligonal, ganglio estrellado, lóbulo cuadrado, pelvis oval, etc.

${ }^{26}$ célula gigante, hipocampo menor, hipocampo mayor, hueso ancho, pelvis pequeña, etc.

${ }_{27}^{27}$ fibra elástica, fibrocartílago esponjoso, pelvis blanda, etc.

${ }^{28}$ marcapasos externo, marcapasos interno, fórceps alto, fórceps bajo, fórceps anterior, etc.

${ }^{29}$ marcapasos permanente, marcapasos provisional, sonda permanente, etc.

${ }^{30}$ electroscopio diferencial, microscopio comparativo, termómetro registrador, etc.

${ }^{31}$ balanza electrodinámica, bisturí eléctrico, electroscopio electrónico, marcapasos nuclear, etc.

${ }^{32}$ balanza automática, microscopio rotatorio, etc.

${ }^{33}$ balanza monoplato, microscopio binocular, etc.

${ }^{34}$ termómetro salino, termómetro metálico, etc.

${ }^{35}$ catéter bicodado, tijeras corvadas, tijeras rectas, etc.

${ }^{36}$ venda elástica, sonda flexible, etc.

${ }_{38}^{37}$ palpación superficial, pelvimetría externa, pelvimetría interna, etc.

38 análisis cruzado, ecografia bidimensional, radiografía panorámica, etc.

${ }^{39}$ análisis funcional, análisis comparado, pelvimetría electiva, etc.

${ }^{40}$ análisis químico, análisis térmico, encefalografia líquida, pelvimetría radiológica, pelvimetría ultrasónica, radiografia neutrónica, etc.

${ }^{41}$ análisis equilibrada, urografía ascendente, etc.

${ }^{42}$ fijación interna, oclusión anterior, oclusión posterior, oclusión lateral, etc.

${ }^{43}$ deshidratación voluntaria, deshidratación accidental, etc. 


$$
\begin{aligned}
& {\left[\text { NOMBRE E }[\mathrm{ADJ} 7]_{\mathrm{SAdj}}\right]_{\mathrm{SN}=\mathrm{UTP}^{4}}{ }^{45}} \\
& {\left[\text { NOMBRE E }[\text { ADJ8 }]_{\text {SAdj }}\right]_{\text {SN }=\text { UTP }}{ }^{46}} \\
& {\left[\text { NOMBRE E }[\text { ADJ } 13]_{\text {SAdj }}\right]_{\mathrm{SN}=\mathrm{UTP}}{ }^{47}} \\
& \text { [NOMBRE E [ADJ15.1 } \left.]_{\mathrm{SAdj}}\right]_{\mathrm{SN}=\mathrm{UTP}}{ }^{48}
\end{aligned}
$$

\section{F. Nombres F:}

$$
\begin{aligned}
& {\left[\text { NOMBRE F }[\text { ADJ1 }]_{\text {SAdj }}\right]_{\mathrm{SN}=\mathrm{UTP}^{40}}{ }^{49}} \\
& \text { [NOMBRE F [ADJ6 } \left.]_{\text {SAdj }}\right]_{\mathrm{SN}=\mathrm{UTP}^{50}} \\
& {\left[\text { NOMBRE F }[\text { ADJ7 }]_{\text {SAdj }}\right]_{\mathrm{SN}}=\mathrm{UTP}^{51}} \\
& \text { [NOMBRE F [ADJ13 } \left.]_{\text {SAdj }}\right]_{\mathrm{SN}}=\mathrm{UTP}^{52} \\
& {\left[\text { NOMBRE F }[\text { ADJ15.4 }]_{\text {SAdj }}\right]_{\mathrm{SN}=\text { UTP }} 53}
\end{aligned}
$$

\section{EN CONCLUSIÓN}

En este artículo hemos querido mostrar el papel decisorio del adjetivo en la discriminación de las UT con estructura $\left[\mathrm{N}[\mathrm{A}]_{\mathrm{SAdj}}\right]_{\mathrm{SN}}$. Primeramente, hemos visto que el adjetivo que integra una UT tiene siempre la función de clasificar al nombre que complementa y motiva que las UT con estructura $\left[\mathrm{N}[\mathrm{A}]_{\mathrm{SAdj}}\right]_{\mathrm{SN}}$ sean siempre un hipónimo de la clase hiperónima que constituye el núcleo nominal de esta unidad. A continuación, hemos analizado los diferentes tipos de informaciones que el adjetivo aporta al nombre en las UT de medicina. Y finalmente hemos propuesto que es justamente la combinación de un tipo de adjetivos con una clase determinada de nombres la que permite la discriminación de las UT con esta estructura y facilita el reconocimiento automático en los casos de ambigüedad.

En este sentido, hemos partido de la idea que un extractor no puede servirse de una única estrategia para detectar las unidades de significación especializada de los textos, sino que se deben aplicar diferentes estrategias según las características internas de cada tipo de unidad. Hemos defendido también que un extractor no puede reducirse a utilizar estrategias que se basen sólo en la forma de las UT poliléxicas. Por eso, para algunas secuencias poliléxicas que son formalmente ambiguas hemos propuesto filtros morfosemánticos basados en esquemas que tienen en cuenta tanto la estructura de una secuencia como la clase semántica de los constituyentes que integran esta secuencia.

${ }^{44}$ coloración protectora, inducción asimiladora, inyección excitante, inyección preservativa, etc.

45 excitación nuclear, inducción electromagnética, inversión eléctrica, inversión química, inversión térmica, etc.

${ }^{46}$ comulsión habitual, nutrición adecuada, oclusión anormal, etc.

${ }^{47}$ convulsión parcial, hospitalización parcial, visión doble, visión múltiple, etc.

${ }^{48}$ hepatización gris, hepatización amarilla, inducción roja, inducción negra, visión verde, etc.

${ }^{49}$ amputación transversal, faringotomía externa, faringotomía interna, indectomía periférica, laringotomía inferior, laringotomía superior, lobotomía frontal, etc.

${ }_{50}$ laparotomía exploradora, iridectomía preparatoria, transfusión substitutiva, etc.

${ }^{51}$ simpactomía química, trasplantación nuclear, etc.

${ }^{52}$ amputación absoluta, iridectomía sectorial, laringotomía completa, laringotomía parcial, mastectomía total, etc.

53 amputación circular, incisión oblicua, incisión transversal, etc. 


\section{BIBLIOGRAFÍA}

BOSQUE, I. Las categorías gramaticales: relaciones y diferencias. Madrid, Síntesis, 1991.

BOSQUE, I. Sobre las diferencias entre los adjetivos relacionales y los calificativos. Revista Argentina de Lingüística, v. 3, p. 10-48, 1993.

CABRÉ, M. T. La terminología: representación y comunicación. Barcelona, Institut Universitari de Lingüística aplicada, UPF, 1999.

CABRÉ, M. T.; ESTOPÀ, R.; VIVALDI, J. Automatic term detection: a review of current system. In: BOURIGAULT, C.; JACQUEMIN, C.; L'HOMME, M-C. (Eds.) Recent advances in Computational Terminology. Amsterdam, John Benjamins, 2000 (en prensa).

ESTOPÀ, R. Les unitats terminológiques polilexemàtiques en els lèxics especialitzats: dret $i$ medicina. Trabajo de investigaicón de doctorado. Barcelona, Institut Universitari de Lingüística Aplicada, UPF, 1996.

ESTOPÀ, R. Extracción de terminología: elementos para la construcción de un SEACUSE (Sistema de Extracción Automática de candidatos a Unidades de Significación Especializada). Barcelona, Institut Universitari de Lingüística Aplicada, UPF, 1999. Tesis de doctorado.

LÓPEZ PIÑERO, J. M.; TERRADA FERRANDIS, M. L. Introducción a la terminología médica. Barcelona, Salvat Editores, 1990.

LORENTE, M. Aspectes de lexicografia: representació $i$ interpretació gramaticals. Barcelona, Universitat de Barcelona, 1994. Tesis de doctorado.

MAINGUENENEAU, D.; SALVADOR, V. Elements de lingüística per al discurs literari. Valencia, Tàndem Edicions, 1995.

PERRON, J. L'analyseur syntaxique: un outil tout à fait adéquat? Terminogramme, 46, p. 29-31, 1988

SAGER, J-C. Curso práctico sobre el procesamiento de la terminología. Madrid, Fundación Germán Sánchez Ruipérez, 1993

SOLER, C. Desajustes léxicos nominales y su representación en una base de conocimiento léxico. Valores semánticos de los adjetivos. Barcelona, Universitat de Barcelona, 1997. Tesis de doctorado.

WIERZBICKA, A. What's in a Noun? (Or: How do Nouns Differ in Meaning from Adjectives?). Studies in Languages, v. 10, n. 2, p. 353-389, 1986. 